Original Article

\title{
Single Institutional Experience with Primary Mediastinal Cysts: Clinicopathological Study of 108 Resected Cases
}

\author{
Yoshiki Kozu, MD, Kenji Suzuki, MD, Shiaki Oh, MD, Takeshi Matsunaga, MD, \\ Yukio Tsushima, MD, and Kazuya Takamochi, MD
}

\begin{abstract}
Purpose: To review our institutional surgical outcomes with primary mediastinal cysts (PMCs) and elucidate the clinicopathological differences among several histological PMC variants.

Methods: We retrospectively reviewed 108 patients who underwent surgery for PMC at our institution between 1997 and 2012.

Results: There were 54 thymic cysts (TCs), 26 bronchogenic cysts (BCs), 16 mature cystic teratomas (MCTs), 11 pericardial cysts (PCs), and 1 esophageal duplication cyst. Surgical approach was via thoracoscopy in 44 , thoracotomy in 24 , median sternotomy in 39 , and hemiclamshell incision in 1. Pathological complete resection was achieved in all patients. Postoperative complications occurred in 13 patients. There was no postoperative mortality. All patients were recurrence-free after a mean follow up of $41 \pm 26$ months. MCT was significantly associated with larger cyst size $(p<0.001)$ more frequent combined resection of invaded organs $(p<0.001)$, more intraoperative bleeding $(p=0.005)$, and longer duration of operation ( $p=0.022$ ) than the 3 other groups (TC, BC, and PC).

Conclusion: Surgical treatment for PMC is safe and efficacious regardless of approach. Patients with MCT may require more aggressive surgeries than those with other histological variants, reflecting their potential for invasion into surrounding structures and larger cyst size.
\end{abstract}

Keywords: primary mediastinal cyst, surgical treatment, histological variant

\section{Introduction}

Primary mediastinal cysts (PMCs) are mainly of embryonal origin, and are uncommon, accounting for approximately $25 \%$ of all mediastinal tumors. ${ }^{1)}$ PMCs encompass a wide variety of histologies, such as thymic

Division of General Thoracic Surgery, Juntendo University School of Medicine, Tokyo, Japan

Received: June 25, 2013; Accepted: August 26, 2013

Corresponding author: Kenji Suzuki, MD. Division of General Thoracic Surgery, Juntendo University School of Medicine, 3-1-3 Hongo, Bunkyo-ku, Tokyo 113-8431, Japan

Email: kjsuzuki@juntendo.ac.jp

(C)2014 The Editorial Committee of Annals of Thoracic and Cardiovascular Surgery. All rights reserved. cyst (TC), bronchogenic cyst (BC), mature cystic teratoma (MCT), pericardial cyst (PC), and esophageal duplication cyst (EDC). With the recent increase in the use of imaging procedures such as computed tomographic (CT) scanning, the chances of encountering this benign entity have increased, even in asymptomatic patients.

Although symptomatic PMC is unequivocally considered indication for surgical treatment, the management of asymptomatic PMC is still debated. Some prefer surgical treatment for fear of malignant transformation, cyst infection, progressive growth, or spontaneous rupture, ${ }^{2-4)}$ whereas others assert that there is no need for universal resection due to the benign nature of PMCs. ${ }^{5-7)}$ Despite this controversy, there has been a paucity of literature concerning surgical experiences with the entire range of PMCs. 
The purpose of the present study was to review our institutional surgical outcomes with PMC over the last 16 years. In addition, we sought to elucidate the differences in clinicopathological features among several histological PMC variants.

\section{Patients and Methods}

We retrospectively reviewed a total of 108 patients who were diagnosed with PMC by histological examination following surgical treatment, at the Juntendo University Hospital, Tokyo, Japan, between 1997 and 2012. We excluded multilocular thymic cysts and cystic thymomas from this study, because the former are associated with thymic neoplasm, ${ }^{8}$ and the latter are occasionally malignant. ${ }^{9)}$

The routine preoperative workup for a mediastinal cystic mass included a complete blood test, chest radiography, and chest CT. Chest magnetic resonance imaging (MRI) and F18-fluorodeoxyglucose positron emission tomography (FDG-PET) were also used when additional radiological information was needed. FDG-PET was available for the last 5 years of this study. Follow up radiological examinations were performed using the following method: chest radiography at every examination in the outpatient department, and chest CT every 6 months.

Medical records of all patients were reviewed in detail regarding gender, age, symptom at presentation, concomitant malignancy, anatomical location of the cystic mass in the mediastinum, ${ }^{10)}$ maximum diameter of the cystic mass on the preoperative CT, surgical approach, extent of surgery, frequency of combined resection of invaded organs, intraoperative bleeding, duration of operation, histological variant, surgical curability, postoperative hospital stay, postoperative complication, mortality, and recurrence. Postoperative mortality was defined as any death during hospitalization or within 30 days from surgery. Approval from the institutional review board was obtained with a waiver for patient consent.

\section{Statistical Analysis}

Of the different histological PMC variant groups, differences were compared using analysis of variance (ANOVA) for continuous variables and $\chi^{2}$ tests for categorical variables. A $p$-value of less than 0.05 was considered significant. The reported $\mathrm{p}$ values are all two-sided. All statistical analyses were performed using JMP 9 software (SAS Institute, Cary, North Carolina, USA).

\section{Results}

There were $52(48 \%)$ male and $56(52 \%)$ female patients. All but 1 ( 9 years old) were adult patients $(>15$ years) with a mean age at surgery of $50 \pm 17$ years. MRI and FDG-PET were used in 72 and 26 patients respectively. FDG-PET was interpreted as positive (maximum standard uptake value $\geq 2.5$ ) in 4 of these 26 . Conversion to open thoracotomy was not required in any patient who underwent thoracoscopic surgery. All thoracoscopic surgeries were performed using 3-4 ports in the lateral position. Histological variants were as follows: $54 \mathrm{TCs}$ (50\%), 26 BCs (24\%), 16 MCTs (15\%), 11 PCs (10\%), and 1 EDC (1\%). Pathological complete resection was achieved in all patients. There was no postoperative mortality. All the patients were recurrence-free after a mean follow up of $41 \pm 26$ months. Clinicopathological features of the patients according to the histological variants are listed in Table $\mathbf{1 .}$

\section{Comparison of clinicopathological features among the 4 groups according to histological PMC variants}

We compared various clinicopathological features among the 4 groups categorized by histological variants, namely TC, BC, MCT and PC (Table 1). We found that there was no significant difference regarding symptoms, concomitant malignancy, postoperative complications, or postoperative hospital stay among the 4 groups. On the other hand, we found that MCT was significantly associated with larger maximum diameter of the cyst $(p<0.001)$, more frequent combined resection of invaded organs $(p<0.001)$, more intraoperative bleeding $(p=0.005)$, and longer duration of operation $(p=0.022)$ than the 3 other groups. Figure 1 shows representative contrastenhanced CT imaging of adhesive cystic teratoma for which combined resection of the lung, mediastinal pleura, and pericardium was required to achieve a complete resection.

\section{Discussion}

This is one of the largest single-center reports on experiences with surgical treatment for PMCs. Although $\mathrm{BC}$ has been reported to be the most common histological variant of $\mathrm{PMC},{ }^{2-4)}$ we found that $\mathrm{TC}$ was the most common in the present study, accounting for half of all cases $(n=54 ; 50 \%)$. The reason for the predominance of $\mathrm{TC}$ in our institution, however, is unclear. 


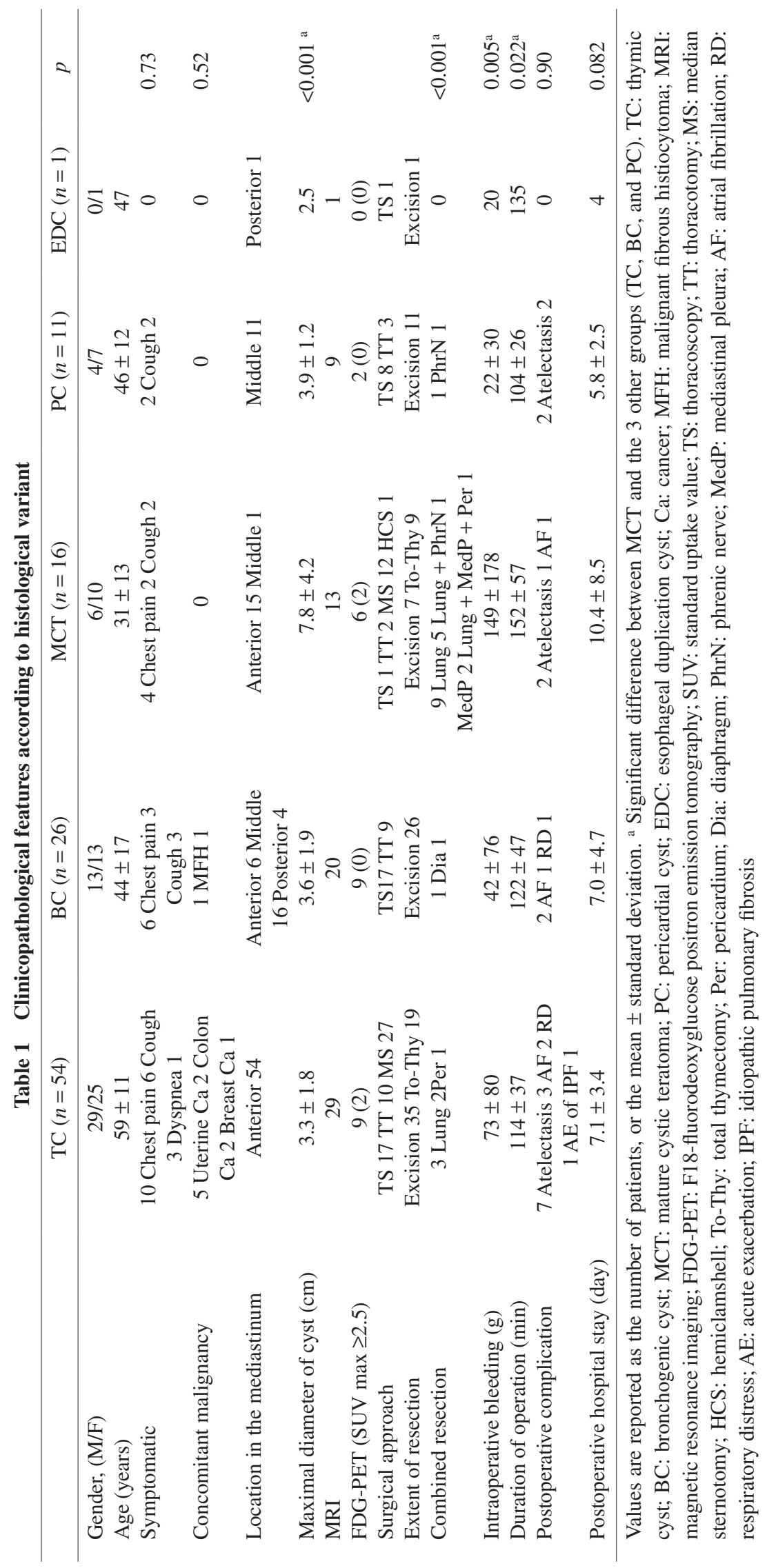




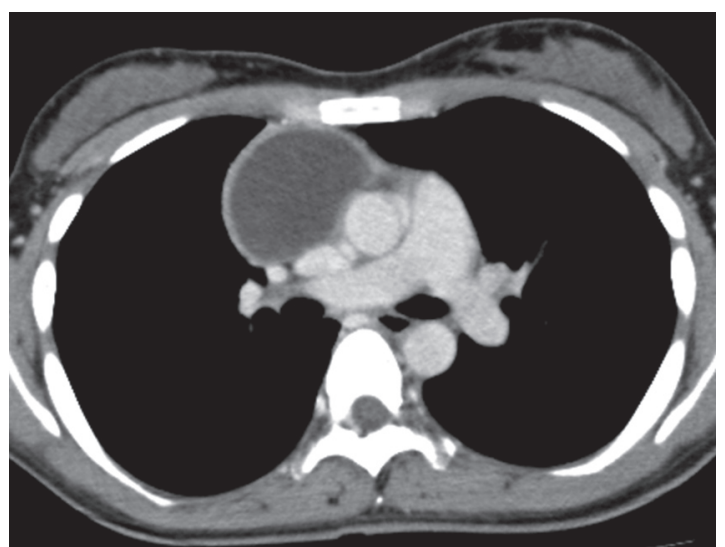

Fig. 1 Representative contrast-enhanced computed tomographic imaging of adhesive cystic teratoma.

In the present study, most of the patients $(n=86 ; 80 \%)$ were asymptomatic. Takeda, et al., ${ }^{3)}$ reported that in a study involving 105 PMC patients, 64\% $(n=67)$ were asymptomatic. They found that those with mesothelial (pericardial/pleural) cysts were more likely to be asymptomatic than those with other histological variants $(p=0.06)$. We, however, detected no correlation between symptomatic status and histological variant $(p=0.73)$. This may be partly because of the small number $(n=22)$ of symptomatic patients.

There is still controversy as to surgical indication for asymptomatic PMC patients. We performed surgery in such patients when malignancy could not be ruled out based on the following radiological features on preoperative $\mathrm{CT}$ and/or MRI: progressive growth, mural nodule, thickened irregular cystic wall, or invasion into the surrounding structures. For the last 5 years of the study period, PET positivity was also considered to be a surgical indication.

We detected a postoperative complication rate of $12 \%$ $(n=13)$, which is comparable to those in previous reports, which range from $6 \%$ to $25 \%$.,4) Postoperative mortality was not observed. Surgical treatment for PMC could be performed safely regardless of the approach used.

Once surgical excision is achieved, postoperative recurrence of PMC is extremely rare. ${ }^{3,4)}$ However, recurrence can occur even 20-25 years after surgery. ${ }^{11,12)}$ Long-term follow up is indicated in case of incomplete resection or intraoperative spillage of the cystic contents due to dense adhesion.

Thoracoscopic surgery for PMC has recently become prevalent, with the advantage of minimal invasiveness. ${ }^{13,14)}$
A thoracoscopic procedure was used in 44 (41\%) patients without conversion to thoracotomy in this cohort. Although this technique offers significant benefits, we should not hesitate to use an open procedure in a complicated case, to achieve complete resection. We could actually obtain pathological complete resection in every case, by using each procedure properly.

The most important clinical implication emerging from this study was that patients with MCT required more aggressive surgeries than those in the 3 other groups (TC, BC, and PC). Specifically, MCT was significantly associated with more frequent combined resection of invaded organs, more intraoperative bleeding, and longer duration of the operation. Although MCT is histopathologically benign as are other histological variants of PMC, MCT can produce proteolytic or digestive enzymes, resulting in rupture and adhesion to other organs. ${ }^{15-17)}$ This potential for invasion into surrounding structures and larger maximum diameter of the cyst may be the explanation for more frequent extended surgeries.

\section{Conclusion}

Surgical treatment for PMC is safe and efficacious regardless of approach. We should keep in mind that MCT may be associated with more aggressive surgeries compared with the other histological variants, reflecting their potential for invasion into surrounding structures and larger cyst size.

\section{Acknowledgement}

We thank Hiroshi Izumi, Division of First Pathology, Juntendo University School of Medicine, for his critical review of the manuscript.

\section{Disclosure Statement}

None declared.

\section{References}

1) Davis RD, Oldham HN, Sabiston DC. Primary cysts and neoplasms of the mediastinum: recent changes in clinical presentation, methods of diagnosis, management, and results. Ann Thorac Surg 1987; 44: 229-37.

2) Esme H, Eren S, Sezer M, et al. Primary mediastinal cysts: clinical evaluation and surgical results of 32 cases. Tex Heart Inst J 2011; 38: 371-4. 
3) Takeda S, Miyoshi S, Minami M, et al. Clinical spectrum of mediastinal cysts. Chest 2003; 124: 125-32.

4) Zambudio AR, Lanzas JT, Calvo MJ, et al. Nonneoplastic mediastinal cysts. Eur J Cardiothorac Surg 2002; 22: 712-6.

5) Bolton JW, Shahian DM. Asymptomatic bronchogenic cysts: what is the best management? Ann Thorac Surg 1992; 53: 1134-7.

6) Fontenelle LJ, Armstrong RG, Stanford W, et al. The asymptomatic mediastinal mass. Arch Surg 1971; 102: 98-102.

7) Ponn RB. Simple mediastinal cysts: resect them all? Chest 2003; 124: 4-6.

8) Suster S, Rosai J. Multilocular thymic cyst: an acquired reactive process. Study of 18 cases. Am J Surg Pathol 1991; 15: 388-98.

9) Graeber GM, Thompson LD, Cohen DJ, et al. Cystic lesion of the thymus. An occasionally malignant cervical and/or anterior mediastinal mass. J Thorac Cardiovasc Surg 1984; 87: 295-300.

10) Shields TW. The mediastinum, its compartments, and the mediastinal lymph nodes. In: Shields TW, LoCicero J III, Ponn RB, et al., eds.; General Thoracic Surgery, 6th ed. Philadelphia: Lippincott Williams \& Wilkins, 2005; 2343.
11) Miller DC, Walter JP, Guthaner DF, et al. Recurrent mediastinal bronchogenic cyst. Cause of bronchial obstruction and compression of superior vena cava and pulmonary artery. Chest 1978; 74: 218-20.

12) Read CA, Moront M, Carangelo R, et al. Recurrent bronchogenic cyst. An argument for complete surgical excision. Arch Surg 1991; 126: 1306-8.

13) De Giacomo T, Diso D, Anile M, et al. Thoracoscopic resection of mediastinal bronchogenic cysts in adults. Eur J Cardiothorac Surg 2009; 36: 357-9.

14) Weber T, Roth TC, Beshay M, et al. Video-assisted thoracoscopic surgery of mediastinal bronchogenic cysts in adults: a single-center experience. Ann Thorac Surg 2004; 78: 987-91.

15) Pikin O, Kolbanov K, Kazakevich V, et al. Mediastinal mature cystic teratoma perforating into the lung. Interact Cardiovasc Thorac Surg 2010; 11: 827-9.

16) $\mathrm{Yu} \mathrm{CW}$, Hsieh MJ, Hwang KP, et al. Mediastinal mature teratoma with complex rupture into the pleura, lung, and bronchus complicated with mycoplasma pneumonia. J Thorac Cardiovasc Surg 2007; 133: 1114-5.

17) Cheung YC, Ng SH, Wan YL, et al. Ruptured mediastinal cystic teratoma with intrapulmonary bronchial invasion: CT demonstration. Br J Radiol 2001; 74: 1148-9. 